\title{
LAMOST PROJECT
}

\author{
Y. $\mathrm{CHU}^{1}$ AND Y-H. ZHAO ${ }^{2}$ \\ ${ }^{1}$ Center for Astrophysics, \\ University of Science and Technology of China, \\ ${ }^{2}$ Beijing Astronomical Observatory, \\ Chinese Academy of Science
}

\section{Introduction}

The Large Sky Area Multi-Object Fiber Spectroscopic Telescope (LAMOST) project is a Chinese National Big Scientific Project. It has been recently been approved by The National Committee of Science and National Committee of Planning. LAMOST will get funds from our government and will start at the end of 1996 . We expect to finish this project within 7 years. Here we describe the LAMOST project briefly.

The Large Sky Area Multi-Object Fibre Spectroscopy Telescope (LAMOST) is a meridian reflecting Schmidt telescope with a clear aperture of 4 -meter, a focal length of 20 -meter and a $5^{\circ}$ field of view. Using active optics to control its reflecting corrector makes LAMOST a unique astronomical instrument in combining a wide field of view with large aperture. The available large linear size focal plane of 1.75 -meter in diameter corresponding to its wide angular field of view of $5^{\circ}$ could accommodate up to 4000 fibers, by which the collected light of celestial objects is fed into the spectrographs, promising a very high spectroscopic acquisition rate, say, of order of ten thousands of spectra per night, or ten million spectra every three years. LAMOST will bring Chinese astronomy into the 21st century with a leading role in the fields of large scale and large sample astronomy and astrophysics.

\section{Design and Performances}

\subsection{GENERAL}

LAMOST is a meridian reflecting Schmidt telescope with the reflecting corrector alt-azimuthally mounted. It consists of seven subsystem, that is, 
optical, mechanical, control, optical fibre positioning, spectrographs and CCD detectors, computer network and telescope enclosure.

Optical system: The main spherical mirror of the Schmidt telescope has a curvature of 40 meters and an area of $6.7 \times 6.02$ meters, consisting of 37 hexagonal segmented mirrors. Its optical axis is fixed in the meridian plane with an angle of 25 degrees to the horizontal plane. The reflecting corrector of the clear aperture of 4 meters is located at the center of the spherical mirror. It consists of 24 hexagonal segmented thin plane mirrors, which are in turn controlled by the active optics system to keep the required aspherical to remove the spherical aberration. The focal length of the optical systeem is 20 meters and its focal ratio of 5 with the field of view of 5 degrees in diameter, thus an angular area of 20 square degrees and a linear size of 1.75 meters in diameter. For more details see the references.

Mechanical structure: A quasi-meridian mounting has been adopted, with a fixed spherical main mirror and an alt-azimuthly mounted reflecting corrector. The focal plane is fixed other than field rotation.

Control System: It is necessary to control the motion of the reflecting corrector in order to track the observed sky area for the 1.5 hours around the meridian and to keep the figure of the corrector as part of an active optics system. Also it controls the precision rotation of the focal plane in order to compensate for the rotation of the field of view.

Optical fibre positioning: There are 4000 optical fibres that require precision placement at the pre-determined positions on the focal plane in order to feed into the slits of the bench spectrographs.

Spectrographs and CCD detectors: They consist of about 20 bench spectrographs with the state of the art CCD detecting systems, including low, intermediate and high spectral resolutions, located in the building underneath the focal plane.

Computer networking: The data acquisition, on-line data handling, data compression, the off-line data procession and so on, are to be connected via a local network.

Telescope enclosure: The design is to take into account the requirement to minimise the degradation of the natural atmospheric seeing by the enclosure and the mirrors, as well as assembly and maintenance.

\subsection{TECHNICAL INNOVATIONS}

The active optics control applied to the reflecting corrector, provides the required real time correction of the spherical aberration during tracking, leading to a combination of large field of view and large clear aperture. In this design, the optical image quality is better than 2 arcseconds (rms $~ 0.7$ arcseconds) at the edge of the field of view (the worst case). 
TABLE 1. Summary of main technical parameters and capability

\begin{tabular}{|c|c|}
\hline \multicolumn{2}{|l|}{ Optical: } \\
\hline Clear aperture & 4 meters \\
\hline Curvature radius & 40 meters \\
\hline Focal length & 20 meters \\
\hline Focal ratio & 5 \\
\hline Field of view & 5 degrees \\
\hline Field of view(linear) & 1.75 meters \\
\hline Image quality & $80 \%$ encircled energy within $1.5-2.0^{\prime \prime}$ \\
\hline \multicolumn{2}{|l|}{ Spectrograph: } \\
\hline Spectral resolution & $10 \AA$ at $5250 \AA$ \\
\hline Spectral range & $3900-9000 \AA$ \\
\hline CCD QE & $50 \%$ \\
\hline CCD dark current & 0.04 electrons sec ${ }^{-1}$ pixel $^{-1}$ \\
\hline CCD readout noise & 5 electrons pixel ${ }^{-1}$ \\
\hline Fibre Diameter & 3.4 arcseconds (330 micrometers) \\
\hline Fibre Cross section & 8.55 square arcseconds \\
\hline \multicolumn{2}{|l|}{ Throughput: } \\
\hline Loss of central obstruction & 0.2 \\
\hline Loss of refelection & 0.3 \\
\hline Loss of optical fibre & 0.2 \\
\hline Loss of spectrograph & 0.75 \\
\hline Atmospheric transmission & 0.8 at $5250 \AA$ \\
\hline Total throughput for object observation & 0.16 \\
\hline Total throughput for sky observation & 0.20 \\
\hline \multicolumn{2}{|l|}{ Observing: } \\
\hline Seeing & 2 arcseconds \\
\hline Additional ground seeing & $1-1.5$ arcseconds \\
\hline Sky brightness & 21 magnitude $\operatorname{arcsec}^{-2}$ at $5250 \AA$ \\
\hline Spectroscopic hours per year & 2000 hours \\
\hline Integration time per observation & 5400 seconds \\
\hline Sky coverage & 24,000 square degrees $-10^{\circ}<\delta<+90^{\circ}$ \\
\hline Limiting magnitudes & 20.5 magnitude at visible \\
\hline Spectra acquisition rate & $10^{7}$ per 3 years (low and int. res.) \\
\hline Predicted Galaxy observations & $2 \times 10^{7}$ \\
\hline Predicted QSO observations & $2 \times 10^{6}$ \\
\hline Predicted Stellar observations & $>5 \times 10^{7}$ \\
\hline
\end{tabular}

The large linear size of the focal plane obtained can easily accommodate 4000 optical fibres, which is unprecedented. 
The segmented mirror concept has been applied to the spherical mirror and the corrector to ease the difficulties in manufacturing large mirrors and lower the cost whilst preventing gravity deformation.

The meridian mounting has been adopted to solve the problem of the layout of long tube (long focal length) corresponding to a large Schmidt telescope. Thus a long focal length is obtained with a large clear aperture, giving a higher spectroscopic performance index $f \Omega$, where $\mathrm{f}$ is the focal length and $\Omega$ is the solid angle of the field of view.

The alt-azimuth mounting has the other advantages, including the fixed focal plane, the fixed optical fibre positioning system and the bench spectrographs, as well as easy accessibility to the optical fibre positioning assembly to change the observed configuration of the objects.

\section{References}

S.-G. Wang, D.-Q. Su, Y.-Q. Chu, Y.-N. Wang and X.-Q. Cui, (1995) "A Special Purpose Schmidt Telescope for Multi-Fibre Astronomical Spetroscopy," in "Wide Field Spectroscopy and the Distant Universe," ed. by S.J. Maddox and A. Aragon-Sabamanca, p. 40, World Scientific Co. Pte. Ltd.

S.-G. Wang, D.-Q. Su, Y.-Q. Chu,X.-Q. Cui and Y.-N. Wang, Applied Optics, 1996, vol.35, No.25, p.5155. 